\title{
MUC2 Expression Is Correlated with Tumor Differentiation and Inhibits Tumor Invasion in Gastric Carcinomas: A Systematic Review and Meta-analysis
}

Jung-Soo Pyo $\cdot$ Jin Hee Sohn Guhyun Kang ${ }^{1}$ · Dong-Hoon Kim Kyungeun Kim · In-Gu Do Dong Hyun Kim

Department of Pathology, Kangbuk Samsung Hospital, Sungkyunkwan University School of Medicine, Seoul; 'Department of Pathology, Inje University Sanggye Paik Hospital, Seoul, Korea

Received: February 25, 2015

Revised: March 23, 2015

Accepted: March 26, 2015

Corresponding Author

Jin Hee Sohn, M.D.

Department of Pathology, Kangbuk Samsung

Hospital, Sungkyunkwan University School of

Medicine, 29 Saemunan-ro, Jongno-gu, Seoul

110-746, Korea

Tel: +82-2-2001-2391

Fax: +82-2-2001-2398

E-mail: jhpath.sohn@samsung.com

\begin{abstract}
Background: While MUC2 is expressed in intestinal metaplasia and malignant lesions, the clinicopathological significance of MUC2 expression is not fully elucidated in gastric carcinoma (GC). Methods: The present study investigated the correlation between MUC2 expression and clinicopathological parameters in 167 human GCs. In addition, to confirm the clinicopathological significance of MUC2 expression, we performed a systematic review and meta-analysis in 1,832 GCs. Results: MUC2 expression was found in 58 of 167 GCs (34.7\%). MUC2-expressing GC showed lower primary tumor $(T)$, regional lymph node $(\mathrm{N})$, and tumor node metastasis $(\mathrm{TNM})$ stages compared with GCs without MUC2 expression ( $p=.001, p=.001$, and $p=.011$, respectively). However, MUC2 expression was not correlated with Lauren's classification and tumor differentiation. In meta-analysis, MUC2 expression was significantly correlated with differentiation and lower tumor stage (odds ratio [OR], 1.303; $95 \%$ confidence interval [Cl], 1.020 to 1.664; $p=.034$ and OR, $1.352 ; 95 \% \mathrm{Cl}, 1.055$ to $1.734 ; \mathrm{p}=.017$, respectively) but not with Lauren's classification, $\mathrm{pN}$ stage, or pTNM stage. Conclusions: MUC2 expression was correlated with a lower tumor depth and lower lymph node metastasis in our study; the meta-analysis showed a correlation of MUC2 expression with tumor differentiation and lower tumor depth.
\end{abstract}

Key Words: Gastric carcinoma; MUC2; Clinicopathological significance; Meta-analysis
Mucin expression shows variable patterns in the gastrointestinal tract based on organ and specific conditions. Gastric markers, such as MUC1, MUC5AC, and MUC6 are expressed in the stomach, and intestinal markers, such as MUC2, may be expressed in cases of intestinal metaplasia or malignant lesions, although MUC2 is not constitutively expressed in normal gastric mucosa. ${ }^{1-4}$ Based on the pattern of intestinal and gastric mucin expression, gastric carcinomas (GCs) are subclassified into gastric, intestinal, mixed, or null phenotypes. ${ }^{4}$ Although many studies have reported the significance of mucin expression and mucin phenotypes and their correlation with tumor behavior and prognosis, the clinicopathological significance has not been fully elucidated in GC.

In gastric mucosa with incomplete intestinal metaplasia (type II or III), which are considered to be precancerous lesions of GC, MUC2 is expressed in both goblet and columnar cells. ${ }^{5,6}$ In ad- dition, MUC2-expressing GCs are believed to result from intestinal metaplasia-dysplasia-carcinoma cascades, ${ }^{5,6}$ although the complete understanding of its regulatory mechanisms and clinicopathological significance is yet to be elucidated.

In the present study, we investigated the correlation between MUC2 expression and clinicopathological parameters in 167 surgically resected GCs using tissue-microarray slides. Systematic review and meta-analysis were performed additionally to confirm the clinicopathological significance of MUC2 expression in all available studies, including the present study.

\section{MATERIALS AND METHODS}

\section{Patients}

The files of 167 patients who had undergone surgical resection of GCs in Kangbuk Samsung Hospital, Sungkyunkwan 
University School of Medicine (Seoul, Korea), from January 1, 1992, to December 31, 1996, were analyzed. We evaluated clinicopathological characteristics, such as age, gender, location of tumor, Lauren's classification, tumor differentiation, lymphatic invasion, nodal metastasis, and pathologic tumor node metastasis (pTNM) stages, by reviewing medical charts, pathological records, and glass slides. The patients had undergone curative resection, subtotal gastrectomy or total gastrectomy. This protocol was reviewed and approved by the Institutional Review Board of Kangbuk Samsung Hospital (approval No. KBC12125).

\section{Tissue array methods}

Seven array blocks containing a total of 167 tissue cores of resected GCs obtained from patients were prepared. Briefly, tissue cores ( $2 \mathrm{~mm}$ in diameter) were taken from individual paraffinembedded GCs (donor blocks) and arranged in recipient paraffin blocks (tissue-array block) using a trephine apparatus. The staining results of the different intra-tumoral areas in these tissue-array blocks showed excellent agreement. A core was chosen from each case for analysis. We defined an adequate case as a tumor occupying more than $10 \%$ of the core area. Each block contained internal controls consisting of non-neoplastic gastric tissue. Sections $4 \mu \mathrm{m}$ in thickness were cut from each tissue-array block, deparaffinized, and dehydrated.

\section{Immunohistochemical staining and evaluation}

Sections were deparaffinized and hydrated by a routine xylenealcohol series. For antigen retrieval, sections were treated with $0.01 \mathrm{M}$ citrate buffer ( $\mathrm{pH}$ 6.0) for 5 minutes in a microwave oven followed by treatment with $3 \% \mathrm{H}_{2} \mathrm{O}_{2}$ to quench endogenous peroxidase. Sections were treated with normal serum of the host animal of the secondary antibody to block nonspecific binding. Sections were then incubated with anti-MUC2 antibody (1:200, Leica Biosystems, Newcastle upon Tyne, UK) as described previously. Immunohistochemical stains were performed using a compact polymer method using a Bond Intense Detection Kit (Leica Biosystems). Visualization was performed by treatment with 3,3'-diaminobenzidine (Vector Laboratories, Burlingame, CA, USA). To confirm the reaction specificity of the antibody, a negative control stain without primary antibody was utilized. All immunostained sections were lightly counterstained with Mayer's hematoxylin.

Immunohistochemical stainings were evaluated by two pathologists. MUC2 showed immunoreactivity in the cytoplasm or cell membrane of tumor cells. Immunostaining results were considered positive if more than $5 \%$ of tumor cells were stained.

\section{Published studies search and selection criteria}

Relevant articles were obtained by searching the PubMed and Web of Science databases up to September 30, 2014. Searches were performed using the following keywords: 'MUC2,' 'gastric carcinoma,' and 'immunohistochemistry.' The title and abstract of all searched articles were screened for exclusion. Review articles were also screened to find additional eligible studies. The search results were then scanned according to the following inclusion and exclusion criteria: (1) MUC2 expression was investigated in human GC tissue, (2) the correlation between MUC2 expression and clinicopathological parameters was included, (3) case reports were excluded, and (4) all were English-language publications.

\section{Data extraction}

Data from all eligible studies were extracted by two pathologists. The following data were extracted from each of the eligible studies: the first author's name, year of publication, manufacturer and dilution ratio of each MUC2 antibody, MUC2 cut-off value, number of patients analyzed, tumor differentiation, and pTNM stage.

\section{Statistical analysis}

Statistical analyses were performed using SPSS ver. 18.0 (SPSS Inc., Chicago, IL, USA). The significance of the correlation between the expression of MUC2 and the clinicopathological parameters was determined by either the $\chi^{2}$ test or the Fisher exact test (two-sided). The results were considered statistically significant when $\mathrm{p}<.05$. Moreover, to perform the meta-analysis, the Comprehensive Meta-Analysis software package (Biostat, Engelwood, NJ, USA) was used. Odds ratios (ORs) with a 95\% confidence interval (CI) were calculated by a fixed-effects model and used to evaluate the correlation between MUC2 expression and clinicopathological parameters. The fixed-effect model was selected in the current meta-analysis, because we analysed correlation between immunoexpression and the clinicopathological parameters by single-effect-event (positive or negative), but not by mean. Heterogeneity between studies was evaluated with the $\mathrm{Q}$ test, $\mathrm{I}^{2}$ statistics and p-values. For assessment of publication bias, Begg's funnel plot and Egger's test were performed. The results were two-sided and considered statistically significant when $\mathrm{p}<.05$.

\section{RESULTS}

\section{Clinicopathological features of MUC2-expressing GCs}

MUC2 was expressed in 58 (34.7\%) of 167 GCs in the im- 
munohistochemical study (Fig. 1). MUC2 expression was significantly higher in early GCs compared to advanced GCs ( $\mathrm{p}=$ .001). In addition, GCs expressing MUC2 showed significantly lower rates of lymphatic invasion, lymph node metastasis, and pTNM stages $(\mathrm{p}=.010, \mathrm{p}=.001$, and $\mathrm{p}=.011$, respectively). However, there was no correlation of MUC2 expression with other clinicopathological parameters such as age, gender, location of tumor, Lauren's classification, or tumor differentiation in our study (Table 1).

\section{Systematic review and meta-analysis}

To confirm the clinicopathological significances of MUC2 expression, we performed a systematic review and meta-analysis. In the current meta-analysis, 195 studies were identified through database searches and were screened. Of these studies, 88 reports were excluded due to insufficient information for correlation between clinicopathological parameters and MUC2 expression. In addition, 32 reports were non-adenocarcinoma studies and 42 reports were non-stomach studies. An additional 22 reports were excluded because they were studies using animal or cell lines, duplicated reports, or case reports. The current meta-analysis included 12 eligible studies, including our data (Fig. 2).,3,8-16 The number of total patients was 1,832 , including 167 patients from our study.

MUC2 expression was found in 827 of 1,832 GCs (45.1\%), and the range in eligible studies was $22.9 \%-90.7 \%$ (Table 2). MUC2 expression was significantly correlated with tumor differentiation (OR, 1.303; 95\% CI, 1.020 to 1.664; $\mathrm{p}=.034 ; \mathrm{I}^{2}=$ 79.3\%) (Fig. 3A) and lower tumor depth (pT1) (OR, 1.352; 95\% CI, 1.055 to $1.734 ; \mathrm{p}=.017 ; \mathrm{I}^{2}=65.7 \%$ ) (Fig. $4 \mathrm{~A}$ ). However, Lauren's classification was not correlated with MUC2 expression (OR, 1.245; $95 \%$ CI, 0.933 to $\left.1.661 ; \mathrm{p}=.137 ; \mathrm{I}^{2}=83.2 \%\right)$ (Fig. 3B), consistent with our immunohistochemical study. Unlike our result, meta-analysis showed no significant correlation between MUC2 expression and nodal stage or TNM stage (OR, $0.872 ; 95 \% \mathrm{CI}, 0.689$ to $1.104 ; \mathrm{p}=.256 ; \mathrm{I}^{2}=68.4 \%$ and $\mathrm{OR}$, 1.208 ; $95 \% \mathrm{CI}, 0.940$ to $1.552 ; \mathrm{p}=.139 ; \mathrm{I}^{2}=21.3 \%$, respectively) (Fig. 4B, C). Significant heterogeneities between studies were identified in tumor differentiation, Lauren's classification, tumor depth and nodal stage but not TNM stage. In Begg's funnel plots,
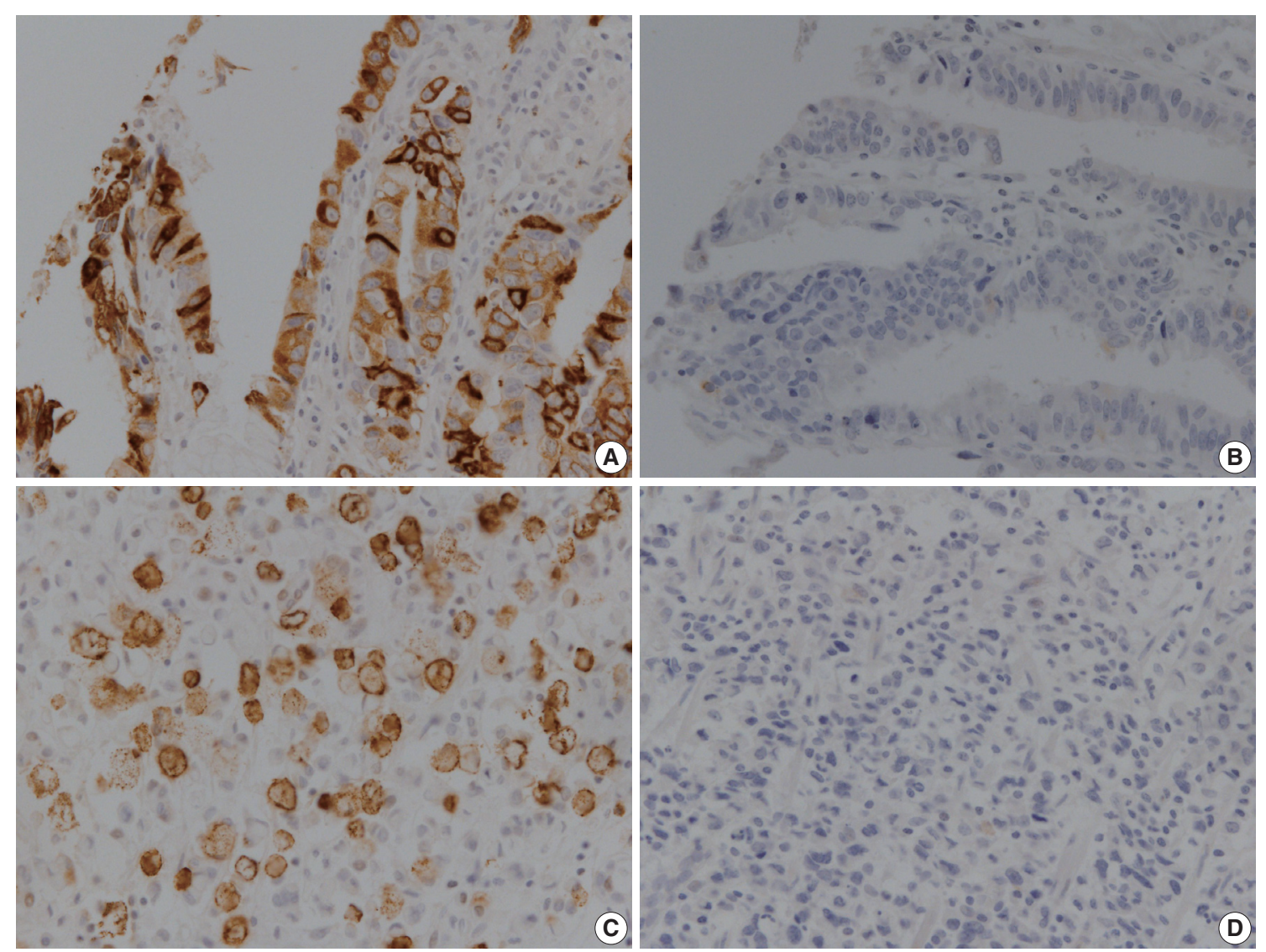

Fig. 1. Representative images showing immunoreactivity for MUC2 in human gastric carcinoma. (A, B) Well-differentiated gastric adenocarcinoma. (C, D) Poorly differentiated gastric adenocarcinoma. 
no definite asymmetry was identified (data not shown). Moreover, Egger's test showed no evidence of publication bias (Table 3). ${ }^{1,3,8-16}$

Table 1. The correlation between the MUC2 expression and clinicopathological parameters in gastric carcinomas

\begin{tabular}{|c|c|c|c|}
\hline Parameter & $\begin{array}{l}\text { MUC2- } \\
\text { negative }\end{array}$ & $\begin{array}{l}\text { MUC2- } \\
\text { positive }\end{array}$ & $p$-value \\
\hline Total $(n=168)$ & 109 (65.3) & $58(34.7)$ & \\
\hline $\begin{array}{c}\text { Age }(y r) \\
0-39 \\
40-65 \\
66-99\end{array}$ & $\begin{array}{l}12(60.0) \\
77(64.2) \\
20(74.1)\end{array}$ & $\begin{array}{r}8(40.0) \\
43(35.8) \\
7(25.9)\end{array}$ & .573 \\
\hline $\begin{array}{l}\text { Gender } \\
\text { Male } \\
\text { Female }\end{array}$ & $\begin{array}{l}77(60.4) \\
32(67.5)\end{array}$ & $\begin{array}{l}37(32.5) \\
21(39.6)\end{array}$ & .365 \\
\hline $\begin{array}{l}\text { Location of tumor } \\
\text { Antrum } \\
\text { Body, cardia }\end{array}$ & $\begin{array}{l}53(62.4) \\
56(68.3)\end{array}$ & $\begin{array}{l}32(37.6) \\
26(31.7)\end{array}$ & .42 \\
\hline $\begin{array}{l}\text { Lauren's classification } \\
\text { Intestinal } \\
\text { Diffuse } \\
\text { Mixed }\end{array}$ & $\begin{array}{r}65(68.4) \\
40(65.6) \\
4(36.4)\end{array}$ & $\begin{array}{r}30(31.6) \\
21(34.4) \\
7(63.6)\end{array}$ & .114 \\
\hline $\begin{array}{l}\text { Tumor differentiation } \\
\text { Well or moderate } \\
\text { Poorly }\end{array}$ & $\begin{array}{l}34(36.6) \\
22(30.6)\end{array}$ & $\begin{array}{l}59(63.4) \\
50(69.4)\end{array}$ & .419 \\
\hline EGC & 28 (48.3) & $30(51.7)$ & .001 \\
\hline AGC & 81 (74.3) & $28(25.7)$ & \\
\hline $\begin{array}{l}\text { Lymphatic invasion } \\
\text { Present } \\
\text { Absent }\end{array}$ & $\begin{array}{l}42(79.2) \\
67(58.8)\end{array}$ & $\begin{array}{l}11(20.8) \\
47(41.2)\end{array}$ & .01 \\
\hline $\begin{array}{l}\text { Lymph node metastasis } \\
\text { Present } \\
\text { Absent }\end{array}$ & $\begin{array}{l}62(77.5) \\
47(54.0)\end{array}$ & $\begin{array}{l}18(22.5) \\
40(46.0)\end{array}$ & .001 \\
\hline $\begin{array}{l}\text { pTNM stage } \\
\text { I } \\
\| \\
\text { III } \\
\text { IV }\end{array}$ & $\begin{array}{r}38(52.8) \\
36(76.6) \\
34(75.6) \\
1(33.3) \\
\end{array}$ & $\begin{array}{r}34(47.2) \\
11(23.4) \\
11(24.4) \\
2(66.7) \\
\end{array}$ & .011 \\
\hline
\end{tabular}

Values are presented as number (\%).

EGC, early gastric carcinoma; AGC, advanced gastric carcinoma.

\section{DISCUSSION}

While various mucins are expressed in GCs, the correlation between MUC2 expression and clinicopathological characteristics remains controversial. In addition, MUC2 could be expressed in benign lesions as well as GC. Therefore, a systemic review and meta-analysis is useful for the elucidation of the clinicopathological significance of MUC2 expression in GCs.

MUC2, an intestinal mucin marker, is not expressed in normal gastric mucosa, ${ }^{17,18}$ unlike other gastric mucin markers such as MUC1, MUC5AC, and MUC6. ${ }^{19}$ The roles of induced MUC2 expression in GC are not fully elucidated and have been controversial in previous studies. In our immunohistochemical study, MUC2 expression was significantly correlated with lower tumor depth $(\mathrm{p}=.001)$, lower nodal metastasis rate $(\mathrm{p}=.001)$, and lower pTNM stage $(\mathrm{p}=.011)$. Unlike our results, the correlations between MUC2 expression and pathologic primary tumor $(\mathrm{pT})$, regional lymph node $(\mathrm{pN})$, and pTNM stage are controversial. $^{1,3,8-16}$ These discrepancies might be caused by various factors, such as composition of tumor types and differences by country. Therefore, it is difficult to determine the clinicopathological significance of MUC2 via our study alone, which led us to analyze previous studies by systematic review and meta-analysis for confirmation of our data.

In the current meta-analysis, MUC2 expression was significantly correlated with lower pT stage, consistent with our results. In our published in vitro study data, MUC2-expressing GC cells showed lower rates of tumor invasion and migration than non-MUC2-expressing GC cells. ${ }^{20}$ This result reinforces the finding that MUC2 expression seems to be associated with lower pT stage. On the other hand, a meta-analysis of colorectal

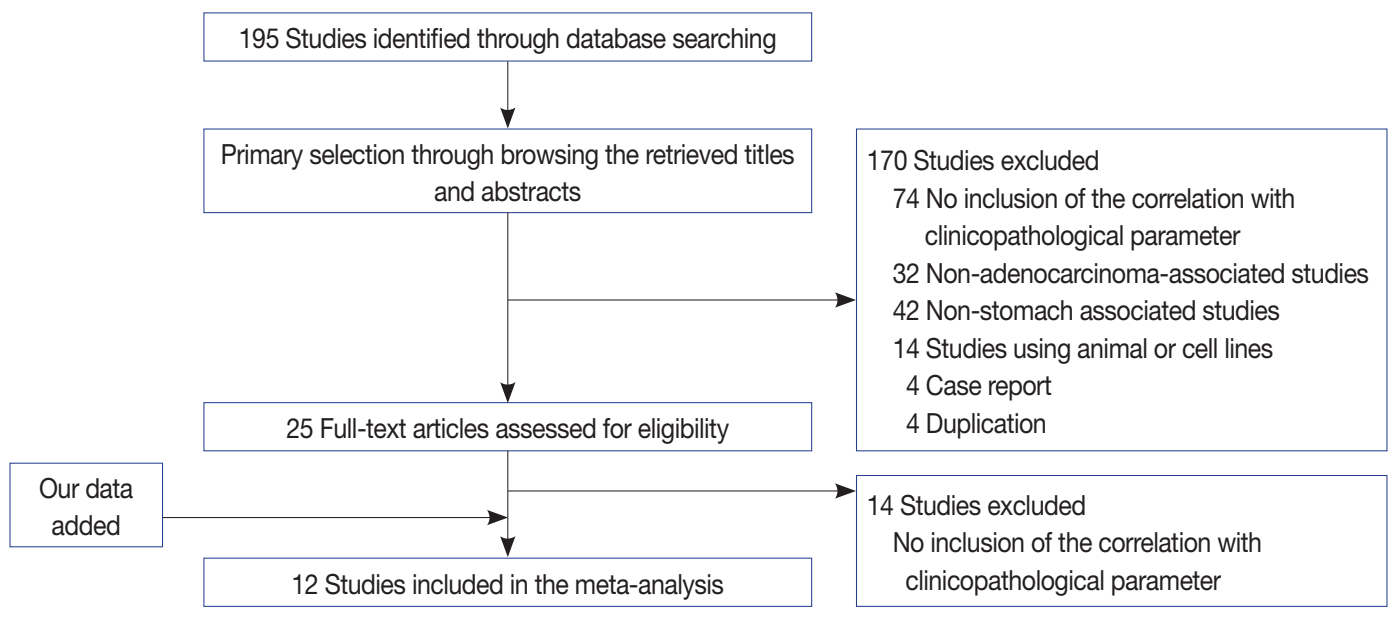

Fig. 2. Flow chart for study search and selection. 
cancer reported that MUC2 positivity was significantly correlated with higher pT3 and pT4 stages. ${ }^{21}$ Correlation with nodal metastasis was controversial in both GC and colon cancer metaanalysis, while our current immunohistochemical study showed a correlation between MUC2 expression and lower nodal metastasis in GC. Further study is needed to define this relationship. Taken together, our results and previous reports suggest that induction of MUC2 may carry out dissimilar functions through different mechanisms according to the specific organ.
Lauren's classification and World Health Organization tumor differentiation are usually used for evaluation of GC in practice. In the current immunohistochemical study, there were no significant correlations between MUC2 expression and Lauren's classification or tumor differentiation. However, the current meta-analysis showed that MUC2 positivity was significantly correlated with degree of GC differentiation, unlike our result. This discrepancy might also be caused by various factors such as composition of tumor types, differences in number of cases analyzed,

Table 2. Main characteristics of the eligible studies

\begin{tabular}{|c|c|c|c|c|c|c|}
\hline Source & Country & Antibody corporation & Dilution ratio & Cut off (\%) & No. of patients & No. of MUC2-positive \\
\hline Akyürek et al. ${ }^{8}$ (2002) & Turkey & Novacastra & $1: 100$ & 5 & 143 & 60 \\
\hline Baldus et al. ${ }^{9}$ (1998) & Germany & Donation & $1: 1,000$ & 5 & 128 & 49 \\
\hline Barresi et al. ${ }^{10}$ (2006) & Italy & Novacastra & $1: 100$ & 5 & 40 & 20 \\
\hline Ilhan et al. ${ }^{1}$ (2010) & Turkey & Neomarkers & $1: 100$ & 5 & 257 & 233 \\
\hline Lee et al. ${ }^{3}$ (2001) & Korea & Santa Cruz & $1: 100$ & 20 & 300 & 82 \\
\hline Lee et al..$^{11}$ (2007) & Korea & Neomarker & - & - & 98 & 53 \\
\hline Shiratsu et al. ${ }^{12}$ (2014) & Japan & Novacastra & $1: 200$ & 5 & 214 & 49 \\
\hline Tanaka et al. ${ }^{13}$ (2003) & Japan & Santa Cruz & $1: 100$ & 30 & 209 & 83 \\
\hline Utsunomiya et al. ${ }^{14}$ (1998) & Japan & Novacastra & $1: 600$ & 5 & 136 & 48 \\
\hline Wang and Fang ${ }^{15}$ (2003) & China & - & $1: 150$ & 10 & 46 & 31 \\
\hline Zhang et al. ${ }^{16}$ (2004) & China & Shenzhen Jingmei Biot & - & - & 94 & 61 \\
\hline Our study & Korea & Novacastra & $1: 100$ & 5 & 167 & 58 \\
\hline
\end{tabular}

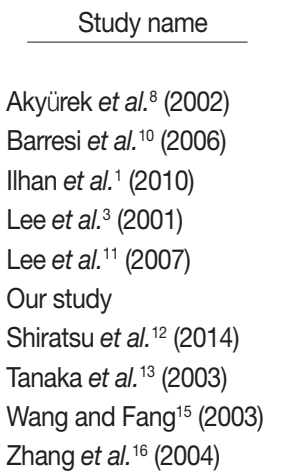

\begin{tabular}{ccc} 
& Statistics for each study \\
\hline Odds ratio & Lower limit & Upper limit \\
1.424 & 0.720 & 2.815 \\
0.076 & 0.016 & 0.358 \\
18.190 & 4.143 & 79.870 \\
0.833 & 0.491 & 1.413 \\
0.621 & 0.238 & 1.619 \\
1.201 & 0.630 & 2.286 \\
0.887 & 0.468 & 1.683 \\
2.287 & 1.246 & 4.198 \\
4.200 & 1.132 & 15.586 \\
4.752 & 1.619 & 13.944 \\
1.303 & 1.020 & 1.664
\end{tabular}

$\mathrm{p}$-value
.309
.001
.000
.499
.330
.578
.714
.008
.032
.005
.034

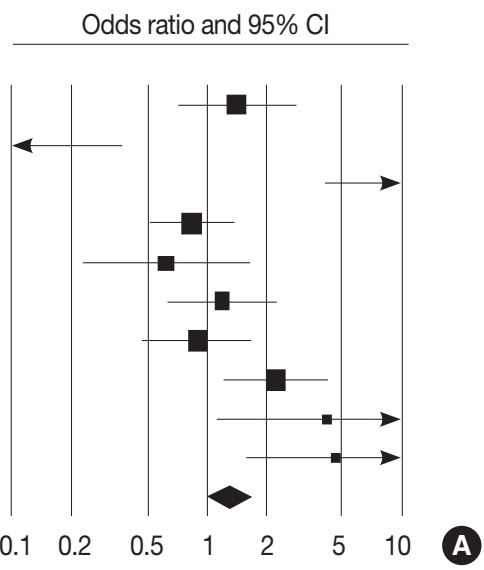

\begin{tabular}{l}
\multicolumn{1}{c}{ Study name } \\
Akyürek et al. ${ }^{8}(2002)$ \\
Baldus et al. ${ }^{9}(1998)$ \\
Barresi et al. ${ }^{10}(2006)$ \\
llhan et al..$^{1}(2010)$ \\
Lee et al. ${ }^{3}(2001)$ \\
Lee et al..$^{11}(2007)$ \\
Our study \\
Wang and Fang ${ }^{15}(2003)$
\end{tabular}

\begin{tabular}{cccc}
\multicolumn{4}{c}{ Statistics for each study } \\
\hline Odds ratio & Lower limit & Upper limit & p-value \\
10.738 & 3.905 & 29.529 & .000 \\
2.651 & 1.252 & 5.614 & .011 \\
0.176 & 0.039 & 0.797 & .024 \\
0.216 & 0.028 & 1.649 & .140 \\
0.692 & 0.408 & 1.176 & .174 \\
2.504 & 1.215 & 5.163 & .013 \\
0.725 & 0.382 & 1.378 & .326 \\
1.071 & 0.314 & 3.655 & .912 \\
1.245 & 0.933 & 1.661 & .137
\end{tabular}

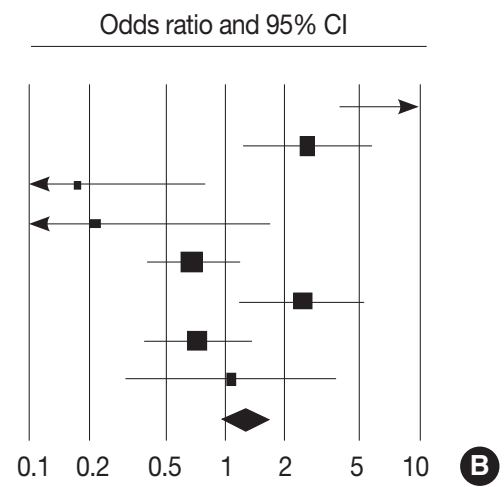

Fig. 3. Forest plot diagram for tumor differentiation (A) and Lauren's classification (B). Cl, confidence interval. 


\begin{tabular}{|c|c|c|c|c|}
\hline \multirow[t]{2}{*}{ Study name } & \multicolumn{4}{|c|}{ Statistics for each study } \\
\hline & Odds ratio & Lower limit & Upper limit & p-value \\
\hline Akyürek et al. ${ }^{8}$ (2002) & 1.670 & 0.571 & 4.889 & .349 \\
\hline Baldus et al. ${ }^{9}$ (1998) & 0.565 & 0.217 & 1.471 & .242 \\
\hline Barresi et al..$^{10}(2006)$ & 0.382 & 0.080 & 1.825 & .228 \\
\hline Lee et al..$^{3}(2001)$ & 1.134 & 0.660 & 1.947 & .648 \\
\hline Lee et al. ${ }^{11}(2007)$ & 0.887 & 0.398 & 1.980 & .770 \\
\hline Our study & 3.099 & 1.585 & 6.061 & .001 \\
\hline Shiratsu et al. ${ }^{12}$ (2014) & 2.090 & 1.089 & 4.013 & .027 \\
\hline Tanaka et al. ${ }^{13}(2003)$ & 1.000 & 0.554 & 1.807 & .999 \\
\hline Utsunomiya et al. ${ }^{14}$ (1998) & 4.118 & 1.582 & 10.720 & .004 \\
\hline Zhang et al. ${ }^{16}$ (2004) & 0.190 & 0.035 & 1.040 & .055 \\
\hline & 1.352 & 1.055 & 1.734 & .017 \\
\hline
\end{tabular}

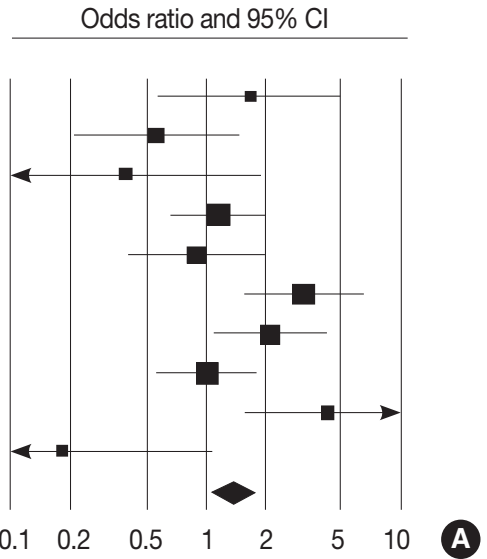

Study name

Akyürek et al. ${ }^{8}$ (2002)

Baldus et al. ${ }^{9}$ (1998)

Barresi et al. ${ }^{10}(2006)$

Illhan et al. ${ }^{1}(2010)$

Lee et al. ${ }^{3}(2001)$

Lee et al. ${ }^{11}(2007)$

Our study

Shiratsu et al. ${ }^{12}$ (2014)

Tanaka et al. ${ }^{13}$ (2003)

Zhang et al. ${ }^{16}(2004)$

\begin{tabular}{cccc}
\multicolumn{4}{c}{ Statistics for each study } \\
\hline Odds ratio & Lower limit & Upper limit & p-value \\
0.677 & 0.326 & 1.406 & .296 \\
0.565 & 0.217 & 1.471 & .242 \\
0.382 & 0.080 & 1.825 & .228 \\
3.901 & 0.891 & 17.081 & .071 \\
1.461 & 0.874 & 2.440 & .148 \\
2.338 & 1.048 & 5.216 & .038 \\
0.341 & 0.174 & 0.669 & .002 \\
1.348 & 0.703 & 2.586 & .368 \\
0.613 & 0.340 & 1.105 & .104 \\
0.470 & 0.199 & 1.113 & .086 \\
0.872 & 0.689 & 1.104 & .256
\end{tabular}

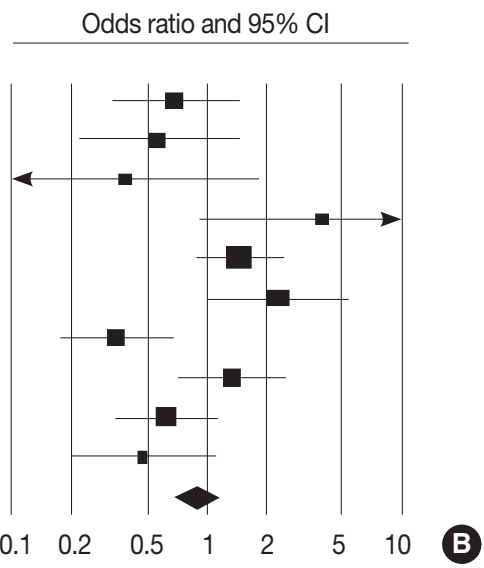

Study name
Akyürek et al..$^{8}(2002)$
Baldus et al..$^{9}(1998)$
Ilhan et al. ${ }^{1}(2010)$
Lee et al. ${ }^{3}(2001)$
Lee et al..$^{11}(2007)$
Our study
Shiratsu et al..$^{12}(2014)$
Tanaka et al..$^{13}(2003)$
Wang and Fang ${ }^{15}(2003)$

\begin{tabular}{cccc}
\multicolumn{4}{c}{ Statistics for each study } \\
\hline Odds ratio & Lower limit & Upper limit & p-value \\
0.654 & 0.324 & 1.323 & .238 \\
1.277 & 0.621 & 2.627 & .506 \\
2.708 & 0.781 & 9.388 & .116 \\
1.032 & 0.616 & 1.729 & .905 \\
2.238 & 0.967 & 5.178 & .060 \\
1.813 & 0.855 & 3.845 & .121 \\
1.466 & 0.731 & 2.940 & .281 \\
1.016 & 0.475 & 2.174 & .967 \\
0.533 & 0.148 & 1.926 & .337 \\
1.208 & 0.940 & 1.552 & .139
\end{tabular}

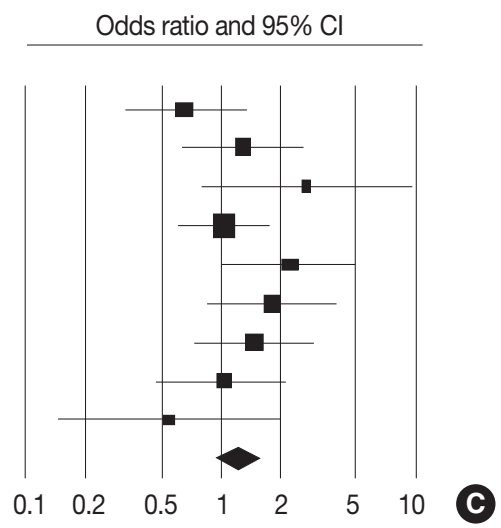

Fig. 4. Forest plot diagram for pathologic primary tumor (A), regional lymph node (B), and tumor node metastasis (C) stages. $\mathrm{Cl}$, confidence interval.

differences by country, and heterogeneity of MUC2 positivity. In addition, whether MUC2 expression is associated with tumor differentiation in GC has not been fully elucidated. Nevertheless, based on previous studies and meta-analysis, MUC2 expression was correlated with differentiation and progression and may be considered to be a representative feature of differentiated cells. ${ }^{14,22}$

In the current meta-analysis, MUC2 expression was shown in
$22.9 \%-90.7 \%$ of GCs. ${ }^{1,3,8-16}$ This wide variability may have been induced by variable dilution ratios and manufacturers of these antibodies. In addition, various cut-off values (5\%-30\%) were used for evaluation of MUC2 expression, and many studies (6 of 11 studies) used a 5\% cut-off value, including ours. MUC2 expression showed significantly higher rates in studies with a $5 \%$ cut-off value than other studies ( $\mathrm{p}<.001$, data not shown). However, the rates of MUC2 expression in subgroups with 5\% 
cut-off value and other cut-off values overlapped at $22.9 \%$ $90.7 \%$ and $27.3 \%-67.4 \%$, respectively. Whether a difference in cut-off value could have an effect on the positive rate of MUC2 expression was not clear in our current systematic review. Moreover, each study included various types of GCs and different compositions. In addition, because some studies used tissue microarray, heterogeneity of MUC2 expression should be considered. These differences may have an effect on the discrepancies of MUC2 positivity between studies. Further studies are needed for confirmation of the clinicopathological significance of MUC2 expression with more eligible criteria and a larger number of patients.

Eligible studies included studies in Korea (3 studies), Japan (3 studies), China (2 studies), Turkey (2 studies), Germany (1 study), and Italy (1 study); eight of twelve studies were conducted in East Asia. According to country, MUC2 positivity was significantly higher in Europe than in East Asia (63.7\% vs. $36.8 \%$, p $<.001$, data not shown). The results of meta-analysis in $\mathrm{pT}$ and $\mathrm{pN}$ stages differed between European and East Asian patients. MUC2 expression was significantly correlated with lower depth of invasion in East Asian patients but not significantly correlated in European patients (OR, 1.484; 95\% CI, 1.134 to 1.941; $\mathrm{p}=$ .004 and $\mathrm{OR}, 0.746 ; 95 \% \mathrm{CI}, 0.448$ to $1.243 ; \mathrm{p}=.261$, respectively). However, there was no correlation between MUC2 expression and lymph node metastasis in both East Asian and European patients. Differences in ethnicity and country may affect the discrepancies in the roles of MUC2 expression. The precise mechanisms are not yet fully understood, and more studies including in vitro study are needed.

In conclusion, our meta-analysis showed that MUC2 expression was significantly correlated with tumor differentiation and depth. However, the correlation between MUC2 expression and other clinicopathological characteristics is controversial. Further studies are needed in order to elucidate the role of MUC2 expression as a prognostic predictor in GC.

\section{Conflicts of Interest}

No potential conflict of interest relevant to this article was reported.

\section{REFERENCES}

1. IIhhan Ö, Han Ü, Önal B, Çelik SY. Prognostic significance of MUC1, MUC2 and MUC5AC expressions in gastric carcinoma. Turk J Gastroenterol 2010; 21: 345-52.

2. Kim JY, Shin NR, Kim A, et al. Microsatellite instability status in gastric cancer: a reappraisal of its clinical significance and relationship with mucin phenotypes. Korean J Pathol 2013; 47: 28-35.

3. Lee HS, Lee HK, Kim HS, Yang HK, Kim YI, Kim WH. MUC1, MUC2, MUC5AC, and MUC6 expressions in gastric carcinomas: their roles as prognostic indicators. Cancer 2001; 92: 1427-34.

4. Wakatsuki K, Yamada Y, Narikiyo M, et al. Clinicopathological and prognostic significance of mucin phenotype in gastric cancer. J Surg Oncol 2008; 98: 124-9.

5. Busuttil RA, Boussioutas A. Intestinal metaplasia: a premalignant lesion involved in gastric carcinogenesis. J Gastroenterol Hepatol 2009; 24: 193-201.

6. Reis CA, David L, Correa P, et al. Intestinal metaplasia of human stomach displays distinct patterns of mucin (MUC1, MUC2, MUC5AC, and MUC6) expression. Cancer Res 1999; 59: 1003-7.

7. Pyo JS, Kang G, Kim DH, et al. Activation of nuclear factor-kappaB contributes to growth and aggressiveness of papillary thyroid carcinoma. Pathol Res Pract 2013; 209: 228-32.

8. Akyürek N, Akyol G, Dursun A, Yamaç D, Günel N. Expression of MUC1 and MUC2 mucins in gastric carcinomas: their relationship with clinicopathologic parameters and prognosis. Pathol Res Pract 2002; 198: 665-74.

9. Baldus $\mathrm{SE}$, Zirbes TK, Engel S, et al. Correlation of the immunohistochemical reactivity of mucin peptide cores MUC1 and MUC2 with the histopathological subtype and prognosis of gastric carcinomas. Int J Cancer 1998; 79: 133-8.

10. Barresi V, Vitarelli E, Grosso M, Tuccari G, Barresi G. Relationship between immunoexpression of mucin peptide cores MUC1 and MUC2 and Lauren's histologic subtypes of gastric carcinomas. Eur J Histochem 2006; 50: 301-9.

11. Lee HW, Yang DH, Kim HK, et al. Expression of MUC2 in gastric carcinomas and background mucosae. J Gastroenterol Hepatol 2007; 22: 1336-43.

12. Shiratsu K, Higuchi K, Nakayama J. Loss of gastric gland mucinspecific O-glycan is associated with progression of differentiatedtype adenocarcinoma of the stomach. Cancer Sci 2014; 105: 126-33.

13. Tanaka M, Kitajima Y, Sato S, Miyazaki K. Combined evaluation of mucin antigen and E-cadherin expression may help select patients with gastric cancer suitable for minimally invasive therapy. Br J Surg 2003; 90: 95-101.

14. Utsunomiya T, Yonezawa S, Sakamoto H, et al. Expression of MUC1 and MUC2 mucins in gastric carcinomas: its relationship with the prognosis of the patients. Clin Cancer Res 1998; 4: 2605-14.

15. Wang RQ, Fang DC. Alterations of MUC1 and MUC3 expression in gastric carcinoma: relevance to patient clinicopathological features. J Clin Pathol 2003; 56: 378-84.

16. Zhang HK, Zhang QM, Zhao TH, Li YY, Yi YF. Expression of mu- 
cins and E-cadherin in gastric carcinoma and their clinical significance. World J Gastroenterol 2004; 10: 3044-7.

17. Audie JP, Janin A, Porchet N, Copin MC, Gosselin B, Aubert JP. Expression of human mucin genes in respiratory, digestive, and reproductive tracts ascertained by in situ hybridization. J Histochem Cytochem 1993; 41: 1479-85.

18. Chang SK, Dohrman AF, Basbaum CB, et al. Localization of mucin (MUC2 and MUC3) messenger RNA and peptide expression in human normal intestine and colon cancer. Gastroenterology 1994; 107: 28-36.

19. Kocer B, Soran A, Kiyak G, et al. Prognostic significance of mucin expression in gastric carcinoma. Dig Dis Sci 2004; 49: 954-64.

20. Pyo JS, Ko YS, Kang G, et al. Bile acid induces MUC2 expression and inhibits tumor invasion in gastric carcinomas. J Cancer Res Clin Oncol 2014 Dec 5 [Epub]. http:/ /dx.doi.org/10.1007/s00432014-1890-1.

21. Li L, Huang PL, Yu XJ, Bu XD. Clinicopathological significance of mucin 2 immuno-histochemical expression in colorectal cancer: a meta-analysis. Chin J Cancer Res 2012; 24: 190-5.

22. Gong L, Debruyne PR, Witek M, et al. Bile acids initiate lineage-addicted gastroesophageal tumorigenesis by suppressing the EGF receptor-AKT axis. Clin Transl Sci 2009; 2: 286-93. 